\title{
N-(Pyridylmethyl)azinium Salts: Precursors of Pyridyl-stabilised Azinium N-Ylides.
}

\author{
Javier Agejas, Ana M. Cuadro, Manuel Pastor, Juan J. Vaquero, José L. García-Navío \\ and Julio A lvarez-B uilla.
}

Departamento de Química Orgánica, Universidad de Alcalá. 28871 Alcalá de Henares. Madrid. Spain.

\begin{abstract}
A series of new N-(pyridylmethyl)azinium salts have been synthesized from 2, 4, 6-triphenylpyrylium tetrafluoroborate. Generation of azinium $\mathrm{N}$-ylides in the presence of base, has been proved by reactions with electrophiles, such as carbon disulphide, phenyl isothiocyanate and with acetylene dipolarophiles.
\end{abstract}

Azinium $N$-ylides are highly interesting compounds due to their reactivity as organic dipoles, as well as to their biological properties and applications. ${ }^{1-3}$ Usually, simple electron-attracting groups, such as carbonyl, ester, cyano etc., have been used to delocalize the negative charge. By contrast, relatively less attention has been paid to the possibility of using heteroaryl groups as stabilizers, except in the case of some tetrazole $^{4 a}$ and triazole-stabilized ylides ${ }^{4 b}$ already described.

Most of our work in the field has been concerned with the synthesis of heteroaryl-stabilized azinium ylides 1 as a way of producing highly stable dipoles, with heterocyclic moieties of unusual reactivity. ${ }^{5}$ More recently, we have been working in the opening of routes to obtain di-heteroaryl $N$-ylides as 2 , and in this paper we report the first synthesis of the corresponding salt precursors, together with the reactivity found for pyridyl-stabilised ylides.

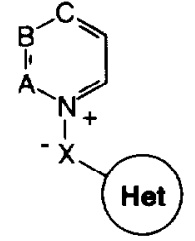

$\mathrm{X}=\mathrm{CH}, \mathrm{N}$

Het= heteroaryi ring

Fig. 1

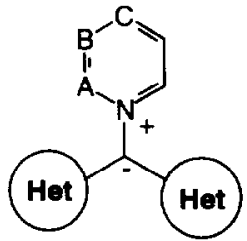

2 


\section{RESULTS AND DISCUSSION}

Synthesis. We had previously described ${ }^{\text {hah }}$ that pyridinium- $N$-(2'-pyridyl)aminides can be prepared from 1-(2',4'-dinitrophenyl)pyridinium halides 4 (Zincke salts). ${ }^{6 c}$ However, adapting this method to the synthesis of the $\mathrm{N}$-ylides 1 and 2 (Route A, Scheme 1) proved to be difficult even in high boiling alcohols, ${ }^{7}$ and yields of salts 9 were not higher than $18 \%$.

As an alternative, we adapted a procedure described by Katritzky ${ }^{8 a}$ to produce, in two steps, the required salts 9 (Route $B$, Scheme 1 ).

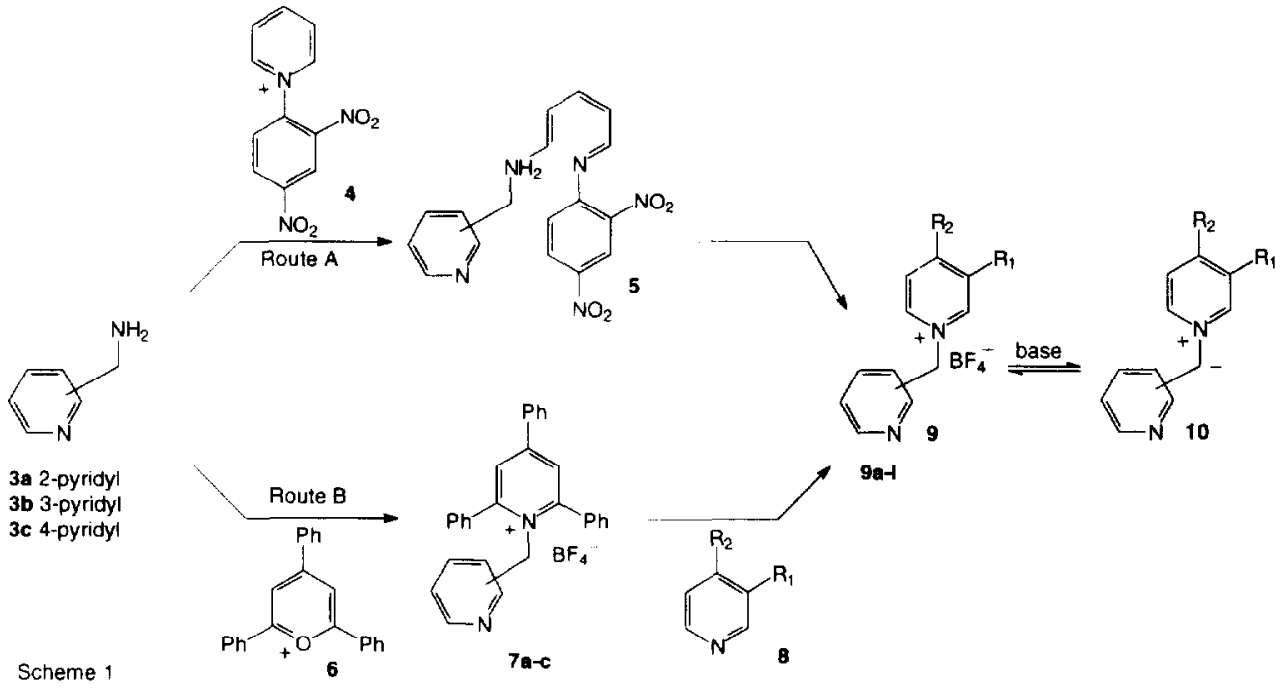

Starting from the aminomethylpyridines 3a-c, the reaction with the 2, 4, 6-triphenylpyrylium salt 6 produced the triphenylpyridinium salts $7 \mathrm{a}-\mathrm{c}^{8 \mathrm{~b}}$ in good yield $(70-74 \%)$ which, when heated with the corresponding azines 8 gave the pyridinium salts $9 \mathrm{a}-1$ (see experimental). This seemed to be an accessible way to produce the pyridinium salts 9 , which on treatment with base, should produce the ylides 10 .

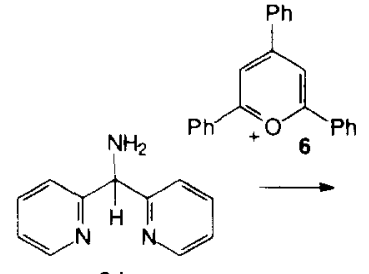

3d<smiles></smiles>

11<smiles>[R2]C1=CC[N+]([CH]C(c2ccccn2)c2ccccn2)(Cc2cnccc2[R2])C=C1[R7]</smiles>

12a-c<smiles>[R6]c1cc[n+](C(c2ccccn2)c2ccccn2)cc1</smiles>

13

Scheme 2

The same procedure was then used to prepare the salt 12 (Scheme 2), starting with di-pyridin-2-yl methylamine 3d. As steric hindrance favours the step 11 to 12 , this should influence the reaction rate. ${ }^{9}$ 
When 3d was treated with the pyrilium salt 6, the hindered 2,4,6-triphenylpyridinium salt 11 was obtained only in $20 \%$ yield. As expected, the nucleophilic displacement of the triphenylpyridine by pyridine, 4 phenylpyridine and isoquinoline, was affected in no more than $30 \mathrm{~min}$, but the expected azinium salts 12 were produced in low yields (10-30\%). Longer reaction times produced extensive decomposition of the final product. The salts 12 on treatment with base should produce the ylides 13 .

Reactivity. Opposite to our experience with related aminides, ${ }^{6 a, b}$ attempts to isolate ylides 10 and 13 had been, up to now, unsuccessful although the formation of ylide 10a, was monitored by ${ }^{1} \mathrm{H}-\mathrm{NMR}$, by observing the deuteration of the methylene group (Scheme 3). Thus, when a solution of $9 \mathrm{a}$ in $\mathrm{CD}_{3} \mathrm{OD}$, in the presence of catalytic concentrations of triethylamine, was maintained at $20 \pm 1^{\circ} \mathrm{C}$ for 7 days, complete exchange of the methylene protons was observed.

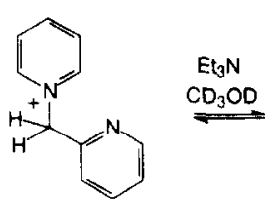

9 a

Scheme 3

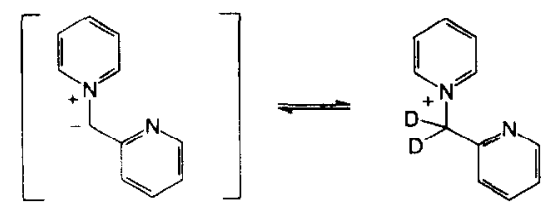

10
14

In a similar way, the salt $\mathbf{1 2 b}$, maintained for 7 days did not produce any trace of deuterated $\mathbf{1 5}$, but was instead converted into the dideuterated derivative 18 , which could have been produced with the assistance of the 2-pyridyl ring nitrogen, as indicated in 16 (Scheme 4). The steric hindrance in 12b should prevent the coplanarity required to stabilise the ylide 13 .

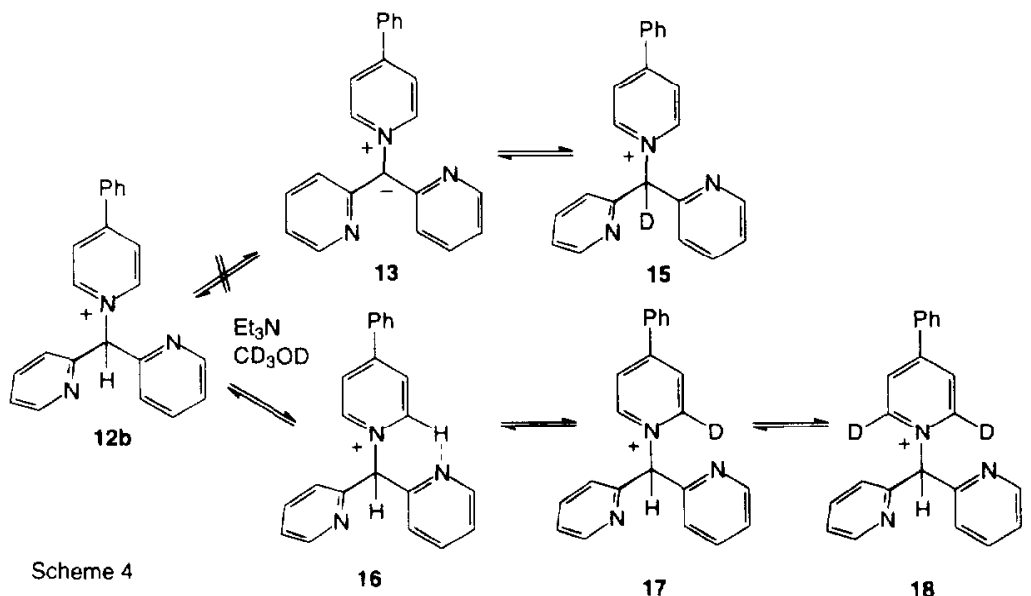

In order to explore the reactivity of ylides $\mathbf{1 0}$ towards electrophiles, we tested the reaction of compounds 9 with halides such as methyl iodide or benzyl chloride in acetone or acetonitrile, in the presence of hase $\left(\mathrm{K}_{2} \mathrm{CO}_{3}\right)$, but no signs of the alkylated derivatives were detected. On the other hand, we tried the substitution of the azine ring by halogenation under mild conditions, as it has been described with related aminides. ${ }^{6 a, b}$ Using either an equimolar amount, or an excess of bromine in the presence of base, in 
dichloromethane at room temperature, extensive decomposition of starting material was observed. The same happened when iodination was tested.

The reaction with acyl halides, as indicated in Scheme 5, produced small yields of the diacylated derivative 20 -presumably through the unstable ylide 19- using two equivalents of the halide. By contrast, when one equivalent was used, only the starting material was recovered.

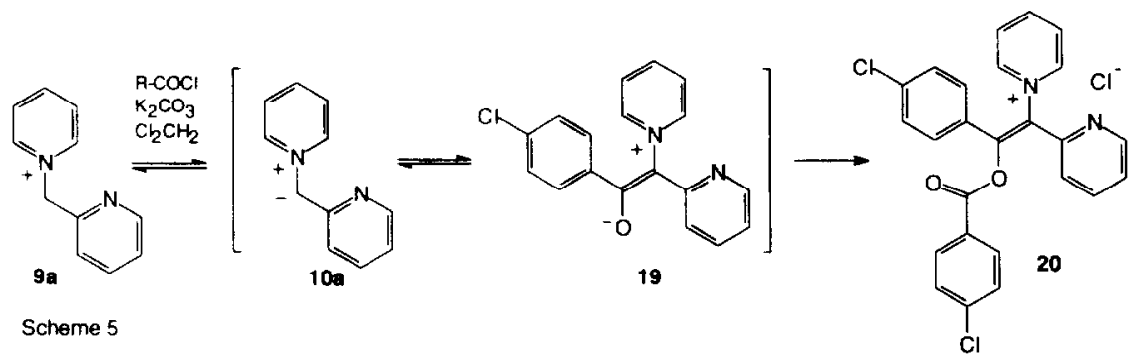

Reaction of pyridinium salts $9 \mathbf{a}, \mathbf{b}$ with dipolarophiles such as dimethyl acetylenedicarboxylate (DMAD) and methyl propiolate in the presence of base, was also tested, and the indolizines 22a-d were obtained in varying yields, according to the expected 1,3-dipolar cycloaddition process (Scheme 6).

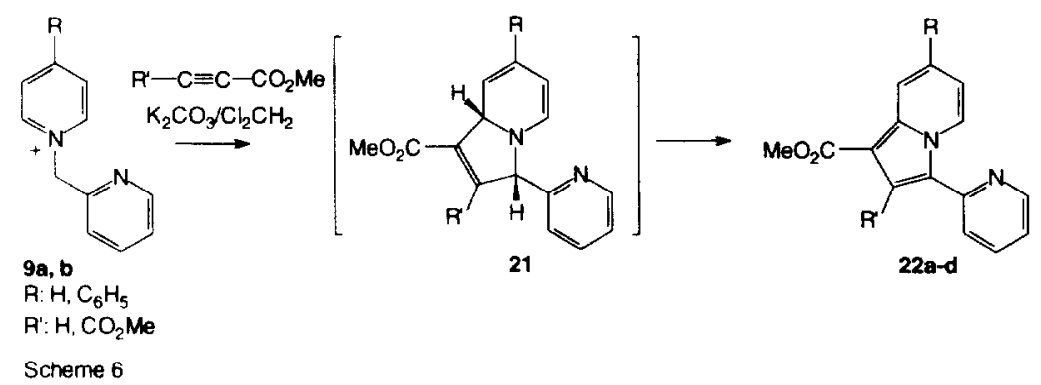

The reactions with heterocumulenes are summarized in Scheme 7. The reaction of 9a with carbon disulphide gave the ketenedithioketal 25 in the presence of $\mathrm{CH}_{3} \mathrm{I}$, as has been previously described

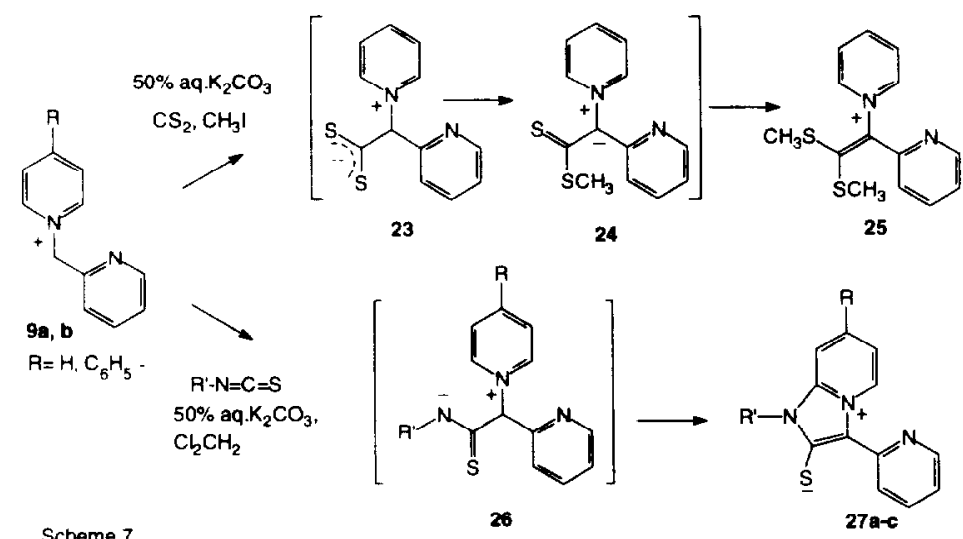

Scheme 7 
with other $\mathrm{N}$-ylides. ${ }^{10}$ The reaction with isothiocyanates, which in biphasic systems normally produces disubstituted ylides, ${ }^{11}$ yielded from 9, the indolizinium thiolates 27 through a 1,3-dipolar cycloaddition, as has been observed previously with ylides stabilised by benzimidazole. ${ }^{12}$ In contrast with the above mentioned results, only the starting material was recovered, from the attempted reaction of $9 \mathbf{a}$ with phenyl isocyanate, using $\mathrm{K}_{2} \mathrm{CO}_{3}$ in acetonitrile.

Theoretical study. With the aim of better understanding the effects preventing the formation of the ylide 13, the conformational differences between the species 10 and 13 were studied. For both species a conformational analysis was performed by systematic rotation of the torsional angles marked as $\alpha, \beta$ and $\gamma$ in figure 2. SPARTAN $3.1^{13}$ was used to systematically rotate each of the angles involved, in $30^{\circ}$ steps (for 10 ) and in $60^{\circ}$ steps (for 13). As $\pi$-delocalization had to be considered to understand the stability of

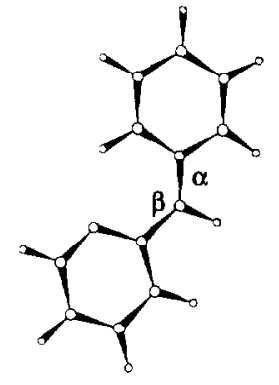

10

Fig.2

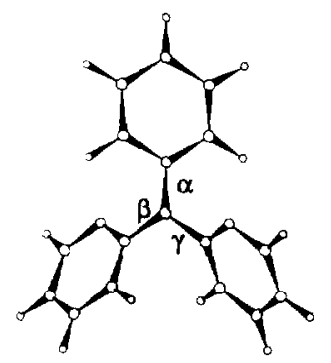

13

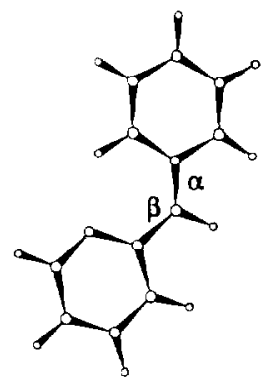

10

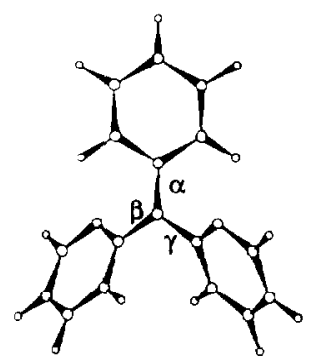

13

the conformers, quantum molecular orbital calculations would produce better results than molecular mechanics. Therefore, the enthalpy of formation of all resulting species was obtained by semiempirical molecular orbital calculations, using AM1 Hamiltonian. The geometries of the more stable conformers, were further optimized, using AM1 as implemented in MOPAC $6.0,{ }^{14}$ until the gradient fell under 0.01 $\mathrm{kcal} / \mathrm{mol}$. Figure 2 represents both optimized geometries.

As it can be seen there, the monosubstituted ylide 10 is flat $\left(\alpha, \beta=0^{\circ}\right)$, confirming an structure stabilised by an efficient intramolecular interaction between the two oppositely charged $\pi$ systems, and by 
a hydrogen bond between $\mathrm{C} 2-\mathrm{H}(\alpha-$ to the pyridinium $\mathrm{N}$ atom) and the 2-pyridyl nitrogen, in a similar way to that described for the analogous aminides. ${ }^{\text {th }}$ By contrast, the disubstituted ylide 13 shows a propellerlike structure $\left(\alpha=31^{\circ}, \beta=35^{\circ}, \gamma=40^{\circ}\right.$ ) due mainly to steric reasons, which prevents the stabilisation. Then, in the presence of base. alternative deuterations were observed (Scheme 4).

As a conclusion, a preparative method for $\mathrm{N}$-(pyridylmethyl)azinium salts $\mathbf{9}$ and $\mathbf{1 2}$ has been developed. The corresponding ylides 10 were generated in the presence of base, and recognized by their reaction products, but they were not isolated, as the related pyridyl-stabilised azinium aminides. ${ }^{6 a, b}$ In contrast, although the dipyridyl salts 12 were obtained, no evidence for the formation of the dipyridylstabilised ylides 13 has been observed.

\section{EXPERIMENTAL}

Melting points were determined on an Electrothermal IA6304 apparatus and are uncorrected. IR spectra were recorded on Perkin-Elmer 700 and 1310 spectrophotometers using KBr pellets. ${ }^{1} \mathrm{H}$-NMR spectra were recorded on a Varian Unity (300 $\mathrm{MHz}$ ) spectrometer. Mass spectra were determined on a Hewlett-Packard 5988A (70 eV) spectrometer. Satisfactory microanalyses were obtained for all new compounds described, within $0.4 \%$ error. Aminomethylpyridines were purchased from Aldrich and used as received. Triphenylpyrylium tetrafluoroborate was prepared as reported. ${ }^{15}$ Silica used for chromatography was 60 Merck, 230-400 mesh.

Reaction of pyrylium tetrafluoroborate with aminomethylpyridines. General procedure. To a stirred suspension of $4 \mathrm{~g}(10 \mathrm{mmol})$ of 2,4,6-triphenylpyrylium tetrafluoroborate in dichloromethane $(50 \mathrm{ml})$, the corresponding aminomethylpyridine $(10 \mathrm{mmol})$ and $1.4 \mathrm{ml}$ of triethylamine $(10 \mathrm{mmol})$ were added, the reaction mixture was stirred for $15 \mathrm{~min}$ and diluted with dry diethyl ether. The resulting precipitate was filtered and recrystallized as indicated.

I.(2- Pyridylmethyl)-2,4,6-triphenylpyridinium tetrafluoroborate (7a). Following the general procedure, using $1.08 \mathrm{~g}(1.05 \mathrm{ml})$ of 2-aminomethylpyridine, $3.6 \mathrm{~g}(74 \%)$ of $7 \mathrm{a}$ as white needles, were isolated. Mp $183-185^{\circ} \mathrm{C}$ (EtOH). IR: $3420,1620,1597,1558,1409,1161,1064,1030 \mathrm{~cm}^{-1} .{ }^{1} \mathrm{H}-\mathrm{NMR}$ (DMSO-d ${ }_{6}$ ) $\delta$ $8,51(\mathrm{~s}, 2 \mathrm{H}), 8.42(\mathrm{dd}, 1 \mathrm{H}, \mathrm{J}=4.9$ and $0.7 \mathrm{~Hz}), 8.28(\mathrm{~d}, 2 \mathrm{H}, \mathrm{J}=6.8 \mathrm{~Hz}), 7.67-7.47(\mathrm{~m}, 14 \mathrm{H}), 7.23(\mathrm{dd}, 1 \mathrm{H}$, $\mathrm{J}=5.4$ and $4.9 \mathrm{~Hz}), 6.68(\mathrm{~d}, 1 \mathrm{H}, \mathrm{J}=7.8 \mathrm{~Hz}), 5.7(\mathrm{~s}, 2 \mathrm{H}) \mathrm{ppm}$. Anal. calcd. for $\mathrm{C}_{29} \mathrm{H}_{23} \mathrm{BF}_{4} \mathrm{~N}_{2}: \mathrm{C}, 71.58$; H, 4.77; N, 5.76. Found: C. $71.28 ;$ H, 4.82: N. 5.62 .

1-(3-Pyridylmethyl)-2,4.6-triphenylpyridinium tetrafluoroborate (7b). Following the general procedure and using $1.08 \mathrm{~g}(1.04 \mathrm{ml})$ of 3 -aminomethylpyridine. $3.4 \mathrm{~g}(70 \%)$ of $7 \mathbf{b}$ as white prisms, were isolated. $\mathrm{Mp}$ $123-124^{\circ} \mathrm{C}(\mathrm{EtOH})$. IR: $3417,3060,1620,1560,1494,1415,1343,1057 \mathrm{~cm}^{-1} .{ }^{1} \mathrm{H}-\mathrm{NMR}\left(\mathrm{DMSO}-\mathrm{d}_{6}\right) \delta$ $8.53(\mathrm{~s}, 2 \mathrm{H}), 8.35-8.26(\mathrm{~m}, 4 \mathrm{H}), 7.74-7.52(\mathrm{~m}, 13 \mathrm{H}), 7.2-7.07(\mathrm{~m}, 2 \mathrm{H}), 5.68(\mathrm{~s}, 2 \mathrm{H}) \mathrm{ppm}$. MS (70 eV) m/e (rel intensity): $399\left(\mathrm{M}^{+}, 2\right), 307(4), 94(100), 80$ (3). Anal. calcd. for $\mathrm{C}_{29} \mathrm{H}_{23} \mathrm{BF}_{4} \mathrm{~N}_{2}: \mathrm{C}, 71.58 ; \mathrm{H}, 4.77 ; \mathrm{N}$, 
5.76. Found: C, $71.24 ; \mathrm{H}, 4.68 ; \mathrm{N}, 5.56$

I-(4-Pyridylmethyl)-2,4,6-triphenylpyridinium tetrafluoroborate (7c). Following the general procedure and using $1.08 \mathrm{~g}(1.04 \mathrm{ml})$ of 4-aminomethylpyridine, $3.6 \mathrm{~g}(74 \%)$ of $7 \mathrm{c}$ as white prisms were isolatcd. Mp $198-200^{\circ} \mathrm{C}(\mathrm{MeOH}) . \mathrm{IR}(\mathrm{KBr}): 3426,1622,1599,1565,1414,1081,1053 \mathrm{~cm}^{-1} .{ }^{1} \mathrm{H}-\mathrm{NMR}\left(\mathrm{DMSO}-\mathrm{d}_{6}\right) \delta$ $8.58(\mathrm{~s}, 2 \mathrm{H}), 8.33-8.27(\mathrm{~m}, 4 \mathrm{H}), 7.70-7.50(\mathrm{~m}, 13 \mathrm{H}), 6.72(\mathrm{~d}, 2 \mathrm{H}, \mathrm{J}=6.1 \mathrm{~Hz}), 5.64(\mathrm{~s}, 2 \mathrm{H})$ ppm. MS $(70$ eV) $\mathrm{m} / \mathrm{e}$ (rel intensity): $399\left(\mathrm{M}^{+}, 3\right), 78(100)$. Anal. calcd. for $\mathrm{C}_{29} \mathrm{H}_{23} \mathrm{BF}_{4} \mathrm{~N}_{2}: \mathrm{C}, 71.58 ; \mathrm{H}, 4.77 ; \mathrm{N}, 5.76$. Found: C, 71.31; H, 4.77; N, 5.71.

Reactions of 1-(2-pyridylmethyl)-2,4,6-triphenylpyridinium tetrafluoroborates 7a-c.

Method A. A solution of the corresponding 1-(2-pyridyl)methyl-2,4,6.-triphenyl pyridinium salt 7 ( 7 mmol) in $15 \mathrm{ml}$ of pyridine, was refluxed for $8-10 \mathrm{~h}$. Then, dry diethyl ether $(3 \times 10 \mathrm{ml})$ was added to the mixture, and the ether fractions were separated. The oily residue was mixed with ether solution of tetrafluoroboric acid $85 \%(1.2 \mathrm{ml}, 7 \mathrm{mmol})$ giving a solid, which was purified by recrystallization as described.

Method B. To a stirred solution of the corresponding triphenylpyridinium salt 7 (2 mmol) in DMF ( $2 \mathrm{ml})$, and the corresponding pyridine $(8 \mathrm{mmol})$ was added, and the reaction mixture was refluxed for the time indicated. Then, the mixture was triturated with diethyl ether $(3 \times 10 \mathrm{ml})$ and the ether extracts were separated. The oily residue was diluted with ethanol $(3 \mathrm{ml})$, ether solution of tetrafluoroboric acid $85 \%$ $(0.4 \mathrm{ml}, 2 \mathrm{mmol})$ was added, and the resulting precipitate was filtered and recrystallized. 1-(2-Pyridylmethyl)pyridinium ditetrafluoroborate $(9 \mathbf{a})$. The method A was used starting from $7 \mathbf{a}(3.4 \mathrm{~g})$ and refluxing the mixture for $10 \mathrm{~h}$. The usual work-up gave $1.3 \mathrm{~g}(55 \%)$ of $9 \mathrm{a}$ as white needles. $\mathrm{Mp} 165$ $166^{\circ} \mathrm{C}$ (EtOH). IR: 3514, 3417, 3033, 2821, 2753, 1634, 1497, $1055 \mathrm{~cm}^{-1}$. 'H-NMR (DMSO-d ' $_{6} \delta 9.12(\mathrm{~d}$, $2 \mathrm{H}, \mathrm{J}=6.4 \mathrm{~Hz}), 8.64(\mathrm{t}, 1 \mathrm{H}, \mathrm{J}=6.6$ and $1.5 \mathrm{~Hz}), 8.47(\mathrm{~d}, 1 \mathrm{H}, \mathrm{J}=4.9 \mathrm{~Hz}, 8.18(\mathrm{t}, 2 \mathrm{H}, \mathrm{J}=6.6 \mathrm{~Hz}), 7.91$ (dt, $1 \mathrm{H}, \mathrm{J}=7.6$ and $1.7 \mathrm{~Hz}), 7.61(\mathrm{~d}, 1 \mathrm{H}, \mathrm{J}=7.8 \mathrm{~Hz}), 7.4(\mathrm{ddd}, 1 \mathrm{H}, \mathrm{J}=7.6,4.9$ and $0.7 \mathrm{~Hz}), 6.0(\mathrm{~s}, 2 \mathrm{H}) \mathrm{ppm}$. MS $(70 \mathrm{eV}) \mathrm{m} / \mathrm{e}$ (rel intensity): $172\left(\mathrm{M}^{+}, 5\right), 94(78), 80(100)$. Anal calcd. for $\mathrm{C}_{11} \mathrm{H}_{12} \mathrm{~B}_{2} \mathrm{~F}_{8} \mathrm{~N}_{2}: \mathrm{C}, 38.29$; H, 3.54; N, 8.16. Found: C, 38.23, H, 3.83; N, 7.93 .

4-Phenyl-1-(2-pyridylmethyl)pyridinium tetrafluoroborate (9b). The method B was used, adding to a solution of $7 \mathrm{c}(0.6 \mathrm{~g}, 1.2 \mathrm{mmol})$ in DMF $(1 \mathrm{ml}), 0.75 \mathrm{~g}(4.8 \mathrm{mmol})$ of 4-phenylpyridine. The mixture was refluxed for $24 \mathrm{~h}$. The usual work-up gave $9 \mathrm{~b}(180 \mathrm{mg}, 45 \%)$ as white prisms, $\mathrm{Mp} 130-132^{\circ} \mathrm{C}$ (EtOH). IR: $3060,1640,1594,1476,1438,1172,1052 \mathrm{~cm}^{1} .{ }^{1} \mathrm{H}-\mathrm{NMR}\left(\mathrm{DMSO}_{-}\right.$) $\delta 9.15(\mathrm{~d}, 2 \mathrm{H}, \mathrm{J}=6.4 \mathrm{~Hz}), 8.60$ $8.50(\mathrm{~m}, 3 \mathrm{H}), 8.08(\mathrm{~d}, 2 \mathrm{H}, \mathrm{J}=5.1 \mathrm{~Hz}), 7.93(\mathrm{t}, 1 \mathrm{H}, \mathrm{J}=7.7 \mathrm{~Hz}), 7.70-7.60(\mathrm{~m}, 4 \mathrm{H}), 7.41(\mathrm{t}, 1 \mathrm{H}, \mathrm{J}=6.2 \mathrm{~Hz})$, $5.99(\mathrm{~s}, 2 \mathrm{H}) \mathrm{ppm}$. MS (70 eV) $\mathrm{m} / \mathrm{e}$ (rel intensity) $247\left(\mathrm{M}^{+}, 4\right), 155(100), 93$ (27). Anal. calcd. for $\mathrm{C}_{17} \mathrm{H}_{15} \mathrm{BF}_{4} \mathrm{~N}_{2}: \mathrm{C}, 61.11 ; \mathrm{H}, 4.52 ; \mathrm{N}, 8.38$. Found: $\mathrm{C}, 60.86 ; \mathrm{H}, 4.41 ; \mathrm{N}, 8.23$.

4-N,N-Dimethylamino-l-(2-pyridy/methyl)pyridinium tetrafluoroborate $(9 \mathrm{c})$. The method $\mathrm{B}$ was used, adding to a stirred solution of $7 \mathrm{a}(0.61 \mathrm{~g}, 1.25 \mathrm{mmol})$ in DMF $(1 \mathrm{ml}), 0.61 \mathrm{~g}(5 \mathrm{mmol})$ of $4-N, N-$

dimethylaminopyridine. The mixture was refluxed for $5 \mathrm{~h}$, then it was allowed to cool, and the usual workup gave 9c (154 mg, 41\%) as white prisms. Mp 136-137 $\mathrm{C}(\mathrm{EtOH}) . \mathrm{IR}: 1653,1569,1435,1402,1224$, 
$1181,1032 \mathrm{~cm}^{-1} .{ }^{1} \mathrm{H}-\mathrm{NMR}\left(\right.$ DMSO-d $\left._{6}\right) \delta 8.5(\mathrm{~d}, 1 \mathrm{H}, \mathrm{J}=4.6 \mathrm{~Hz}), 8.32(\mathrm{~d}, 2 \mathrm{H}, \mathrm{J}=7.8 \mathrm{~Hz}), 7.86(\mathrm{dt}, 1 \mathrm{H}, \mathrm{J}=$ 7.6 and $1.7 \mathrm{~Hz}), 7.45(\mathrm{~d}, 1 \mathrm{H}, \mathrm{J}=7.8 \mathrm{~Hz}), 7.36(\mathrm{dd}, 1 \mathrm{H}, \mathrm{J}=7.6$ and $4.9 \mathrm{~Hz}), 7.04(\mathrm{~d}, 2 \mathrm{H}, \mathrm{J}=7.6 \mathrm{~Hz}), 5.51$ (s, 2II), 3.17 (s, 6H) ppm. Anal. calcd. for $\mathrm{C}_{13} \mathrm{H}_{16} \mathrm{BF}_{4} \mathrm{~N}_{3}: \mathrm{C}, 51.86 ; \mathrm{H}, 5.35 ; \mathrm{N}, 13.95$. Found: C, 51.96; $\mathrm{H}, 5.18 ; \mathrm{N}, 13.86$.

I-(2-Pyridylmethyl)isoquinolinium ditetrafluoroborate (9d). Following the method B, compound $7 \mathbf{a}(0.93 \mathrm{~g}$, $2 \mathrm{mmol})$ and isoquinoline $(1.03 \mathrm{~g}, 0.94 \mathrm{ml}, 8 \mathrm{mmol})$ were refluxed for $24 \mathrm{~h}$. The usual work-up yielded 9d $(80 \mathrm{mg}, 10 \%)$ as white prisms. Mp $153-155^{\circ} \mathrm{C}(\mathrm{EtOH}) . \mathrm{IR}: 3485,3012,2692,1643,1470,1400,1056 \mathrm{~cm}^{-}$

l. ${ }^{1} \mathrm{H}-\mathrm{NMR}\left(\right.$ DMSO-d $\left._{6}\right) \delta 10.18(\mathrm{~s}, 1 \mathrm{H}), 8.79(\mathrm{~d}, 1 \mathrm{H}, \mathrm{J}=6.8 \mathrm{~Hz}), 8.59(\mathrm{~d}, 1 \mathrm{H}, \mathrm{J}=6.8 \mathrm{~Hz}), 8.54(\mathrm{~d}, 1 \mathrm{H}, \mathrm{J}=$ $8.3 \mathrm{~Hz}), 8.46(\mathrm{~d} .1 \mathrm{H}, \mathrm{J}=4.9 \mathrm{~Hz}), 8.35(\mathrm{~d} .1 \mathrm{H} . \mathrm{J}=8.1 \mathrm{~Hz}), 8.28(\mathrm{dd}, 1 \mathrm{H}, \mathrm{J}=8.1$ and $7.1 \mathrm{~Hz}), 8.08(\mathrm{t}, 1 \mathrm{H}$, $\mathrm{J}=7.5 \mathrm{~Hz}) 7.92(\mathrm{dt}, 1 \mathrm{H}, \mathrm{J}=7.8$ and $1.7 \mathrm{~Hz}), 7.68(\mathrm{~d}, 1 \mathrm{H}, \mathrm{J}=7.8 \mathrm{~Hz}), 7.40(\mathrm{dd}, 1 \mathrm{H}, \mathrm{J}=7.6$ and $4.9 \mathrm{~Hz})$, $6.12(\mathrm{~s}, 2 \mathrm{H}) \mathrm{ppm}$. MS (70 eV) m/e (rel intensity) $221\left(\mathrm{M}^{+}, 8\right), 130(100), 94$ (48). Anal. calcd. for $\mathrm{C}_{15} \mathrm{H}_{14} \mathrm{~B}_{2} \mathrm{~F}_{8} \mathrm{~N}_{2}: \mathrm{C}, 45.51 ; \mathrm{H}, 3.56 ; \mathrm{N}, 7.08$. Found: C, 45.22; H, 3.50; N, 6.87.

1-(3-Pyridylmethyl)pyridinium ditetrafluoroborate (9e). Following the method A, $7 \mathbf{b}(0.52 \mathrm{~g}, 1 \mathrm{mmol})$ and pyridine $(2.5 \mathrm{ml}, 30 \mathrm{mmol})$ were refluxed for $8 \mathrm{~h}$. The usual work-up gave $9 \mathrm{e}(100 \mathrm{mg}, 30 \%)$ as white plates. Mp $162-164^{\circ} \mathrm{C}(\mathrm{MeOH})$. IR: $3411,3032,2732,1632,1555,1494,1300,1055 \mathrm{~cm}^{-1}$; ${ }^{1} \mathrm{H}-\mathrm{NMR}$ $\left(\mathrm{DMSO}-\mathrm{d}_{6}\right) \delta 9.18(\mathrm{~d}, 2 \mathrm{H}, \mathrm{J}=6.1 \mathrm{~Hz}), 8.99(\mathrm{~d}, 1 \mathrm{H}, \mathrm{J}=2.0 \mathrm{~Hz}), 8.84(\mathrm{dd}, 1 \mathrm{H}, \mathrm{J}=5.4$ and $1.2 \mathrm{~Hz}), 8.63(\mathrm{t}$, $1 \mathrm{H}, \mathrm{J}=7.8 \mathrm{~Hz}), 8.40(\mathrm{dd}, 1 \mathrm{H}, \mathrm{J}=8.0$, and $1.7 \mathrm{~Hz}), 8.19(\mathrm{t}, 2 \mathrm{H}, \mathrm{J}=7.2 \mathrm{~Hz}), 7.87(\mathrm{dd}, 1 \mathrm{H}, \mathrm{J}=8.1$ and 5.4 $\mathrm{Hz}), 5.96(\mathrm{~s}, 2 \mathrm{H}) \mathrm{ppm}$; MS (70 eV) m/e (rel intensity): $172\left(\mathrm{M}^{+}, 8\right), 94(89), 80(100)$; Anal. calcd. for $\mathrm{C}_{11} \mathrm{H}_{12} \mathrm{~B}_{2} \mathrm{~F}_{8} \mathrm{~N}_{2}: \mathrm{C}, 38.29 ; \mathrm{H}, 3.54 ; \mathrm{N}, 8.16$. Found: C, 38.37; H, 3.60; N, 8.26.

4-Phenyl-1-(3-pyridylmethyl)pyridinium tetrafluoroborate ( $9 \mathrm{f})$. According to method $\mathrm{B}$, to a solution of $7 \mathrm{c}$ $(0.3 \mathrm{~g}, 0.6 \mathrm{mmol})$ in DMF $(1 \mathrm{ml}), 0.38 \mathrm{~g}$ (2.4 mmol) of 4-phenylpyridine were added, and the mixture was refluxed for $6 \mathrm{~h}$, and the usual work-up yielded $9 \mathrm{f}(100 \mathrm{mg}, 50 \%)$ as white prisms. Mp 149-150 $\mathrm{C}$ (EtOH). IR: 3060, 1638, 1434, 1166, $1059 \mathrm{~cm}^{-1} .{ }^{1} \mathrm{H}-\mathrm{NMR}\left(\mathrm{DMSO}_{-\mathrm{d}_{6}}\right) \delta 9.23(\mathrm{~d}, 2 \mathrm{H}, \mathrm{J}=7.1 \mathrm{~Hz}), 8.82(\mathrm{~s}, 1 \mathrm{H})$, $8.62(\mathrm{~d}, 1 \mathrm{H}, \mathrm{J}=4.6 \mathrm{~Hz}), 8.53(\mathrm{~d}, 2 \mathrm{H}, \mathrm{J}=7.1 \mathrm{~Hz}), 8.10-8.05(\mathrm{~m}, 2 \mathrm{H}), 7.99(\mathrm{~d}, 1 \mathrm{H}, \mathrm{J}=7.8 \mathrm{~Hz}), 7.63(\mathrm{~m}$, $3 \mathrm{H}), 7.47(\mathrm{dd}, 1 \mathrm{H}, \mathrm{J}=7.8$ and $4.9 \mathrm{~Hz}), 5.86(\mathrm{~s}, 2 \mathrm{H}) \mathrm{ppm}$. MS $(70 \mathrm{eV}) \mathrm{m} / \mathrm{e}$ (rel intensity) $247\left(\mathrm{M}^{+}, 16\right)$, $156(100), 94$ (33). Anal. calcd. for $\mathrm{C}_{17} \mathrm{H}_{15} \mathrm{BF}_{4} \mathrm{~N}_{2}: \mathrm{C}, 61.11 ; \mathrm{H}, 4.52 ; \mathrm{N}, 8.38$.Found: $\mathrm{C}, 60.98 ; \mathrm{H}, 4.28 ; \mathrm{N}$, 8.27

4-N,N-Dimethylamino-I-(3-pyridylmethyl)pyridinium tetrafluoroborate $(\mathbf{9 g})$. According to method $\mathbf{B}$, to a solution of $7 \mathrm{~b}(0.8 \mathrm{~g}, 1.6 \mathrm{mmol})$ in DMF $(1 \mathrm{ml}), 0.8 \mathrm{~g}(6.4 \mathrm{mmol})$ of $4-N, N$-dimethylaminopyridine were added. The mixture was refluxed for $3 \mathrm{~h}$, and the usual work-up gave $9 \mathrm{~g}(0.35 \mathrm{~g}, 47 \%)$ as white prisms. Mp 98-100 ${ }^{\circ} \mathrm{C}(\mathrm{EtOH})$. IR: 3423, 1650, 1573, 1432, 1404, 1169, $1059 \mathrm{~cm}^{-1} .{ }^{1} \mathrm{H}-\mathrm{NMR}$ (DMSO-d ${ }_{6}$ ) $\delta 8.65$ (d, $1 \mathrm{H}, \mathrm{J}=2.2 \mathrm{~Hz}), 8.56(\mathrm{dd}, 1 \mathrm{H}, \mathrm{J}=4.8$ and $1.5 \mathrm{~Hz}), 8.40(\mathrm{~d}, 2 \mathrm{H}, \mathrm{J}=7.7 \mathrm{~Hz}), 7.8(\mathrm{dt}, 1 \mathrm{H}, \mathrm{J}=7.9$ and 1.9 $\mathrm{Hz}), 7.42(\mathrm{dd}, 1 \mathrm{H}, \mathrm{J}=7.7$ and $4.8 \mathrm{~Hz}), 7.03(\mathrm{~d}, 2 \mathrm{H}, \mathrm{J}=7.7 \mathrm{~Hz}), 5.41(\mathrm{~s}, 2 \mathrm{H}), 3.16(\mathrm{~s}, 6 \mathrm{H}) \mathrm{ppm}$. MS $(70$ eV) m/e (rel. intensity) $214\left(\mathrm{M}^{+}, 3\right), 123(100), 94$ (96). Anal. calcd. for $\mathrm{C}_{13} \mathrm{H}_{16} \mathrm{BF}_{4} \mathrm{~N}_{3}: \mathrm{C}, 51.86 ; \mathrm{H}, 5.35$; N, 13.95. Found: C, 51.65; H, 5.15; N, 13.80. 
I-(3-Pyridylmethyl)isoquinolinium ditetrafluoroborate $(9 \mathrm{~h})$. Following the procedure B, compound $7 \mathrm{~b}(0.93$ $\mathrm{g}, 2 \mathrm{mmol})$ and isoquinoline $(1.03 \mathrm{~g}, 0.94 \mathrm{ml}, 8 \mathrm{mmol})$ were refluxed for $6 \mathrm{~h}$. The usual work-up gave palc orange prisms of $9 \mathrm{~h}(0.45 \mathrm{~g}, 57 \%)$. Mp $162-164^{\circ} \mathrm{C}$ (EtOH). IR: $3409,3103,1640,1611,1553,1470$, 1447, 1385, 1289, $1048 \mathrm{~cm}^{-1}$. ${ }^{1} \mathrm{H}-\mathrm{RMN}\left(\mathrm{DMSO}_{6}\right) \delta 10.20(\mathrm{~s}, 1 \mathrm{H}), 9.0(\mathrm{~s}, 1 \mathrm{H}), 8.80(\mathrm{~m}, 2 \mathrm{H}), 8.59(\mathrm{~d}, 1 \mathrm{H}$, $\mathrm{J}=6.8 \mathrm{~Hz}), 8.50(\mathrm{~d}, 1 \mathrm{H}, \mathrm{J}=8.1 \mathrm{~Hz}), 8.40-8.20(\mathrm{~m}, 3 \mathrm{H}), 8.08(\mathrm{t}, 1 \mathrm{H}, \mathrm{J}=7.6 \mathrm{~Hz}), 7.78(\mathrm{dd}, 1 \mathrm{H}, \mathrm{J}=8.0$ and $5.4 \mathrm{~Hz}), 6.07$ (s, 2H) ppm. MS (70 eV) m/e (rel intensity) $222\left(\mathbf{M}^{+}, 7\right), 130$ (100), 94 (8). Anal. calc. for $\mathrm{C}_{15} \mathrm{H}_{14} \mathrm{~B}_{2} \mathrm{~F}_{8} \mathrm{~N}_{2}: \mathrm{C}, 45.51 ; \mathrm{H}, 3.56 ; \mathrm{N}, 7.08$. Found: $\mathrm{C}, 45.80 ; \mathrm{H}, 3.64 ; \mathrm{N}, 7.31$.

1-(4-Pyridylmethyl)pyridinium ditetrafluoroborate (9i). Following the method A, $7 \mathrm{c}(1.0 \mathrm{~g}, 2 \mathrm{mmol})$ and pyridine $(5 \mathrm{ml}, 60 \mathrm{mmol})$ were refluxed for $8 \mathrm{~h}$. The usual work-up gave $210 \mathrm{mg}$ of $9 \mathrm{i}$ as pale orange prisms $(30 \%)$. Mp $187-188^{\circ} \mathrm{C}(\mathrm{MeOH})$. IR: $3281,3099,3026,1639,1607,1496,1299,1054 \mathrm{~cm}^{-1} .{ }^{1} \mathrm{H}-$ NMR $(300 \mathrm{MHz} \text {, DMSO-d })_{6} \delta 9.16(\mathrm{~d}, 2 \mathrm{H}, \mathrm{J}=6.6 \mathrm{~Hz}), 8.81(\mathrm{~d}, 2 \mathrm{H}, \mathrm{J}=5.1 \mathrm{~Hz}), 8.70(\mathrm{t}, 1 \mathrm{H}, \mathrm{J}=7.9 \mathrm{~Hz})$, $8.24(\mathrm{t}, 2 \mathrm{H}, \mathrm{J}=7.8 \mathrm{~Hz}), 7.72(\mathrm{~d}, 2 \mathrm{H}, \mathrm{J}=4.9 \mathrm{~Hz}), 6.05(\mathrm{~s}, 2 \mathrm{H}) \mathrm{ppm}$. MS $(70 \mathrm{eV}) \mathrm{m} / \mathrm{e}$ (rel intensity) 171 $\left(\mathrm{M}^{+}, 3\right), 94$ (24), 80 (100). Anal.calcd. for $\mathrm{C}_{11} \mathrm{H}_{12} \mathrm{~B}_{2} \mathrm{~F}_{8} \mathrm{~N}_{2}: \mathrm{C}, 38.29 ; \mathrm{H}, 3.54 ; \mathrm{N}, 8.16$; Found:C, 38.29; $\mathrm{H}$, $3.56 ; \mathrm{N}, 8.08$.

4-Phenyl-1-14-Pyridylmethyl)pyridinium tetrafluoroborate $(9 \mathbf{j})$. According to method $\mathrm{B}$, to a solution of $0.6 \mathrm{~g}(1.2 \mathrm{mmol})$ of $7 \mathbf{b}$ in DMF $(2 \mathrm{ml}), 0.75 \mathrm{~g}(4.8 \mathrm{mmol})$ of 4-phenylpyridine were added, and the mixture was refluxed for $6 \mathrm{~h}$. The usual work-up gave $9 \mathrm{j}(160 \mathrm{mg}, 40 \%)$ as white prisms. Mp 196$197^{\circ} \mathrm{C}(\mathrm{EtOH})$. IR: $1639,1599,1491,1441,1418,1209,1182,1060 \mathrm{~cm}^{-1} .{ }^{1} \mathrm{H}-\mathrm{NMR}$ (DMSO-d $) \delta 9.19(\mathrm{~d}$, $2 \mathrm{H}, \mathrm{J}=5.6 \mathrm{~Hz}), 8.63(\mathrm{~d}, 2 \mathrm{H}, \mathrm{J}=4.4 \mathrm{~Hz}), 8.57(\mathrm{~d}, 2 \mathrm{H}, \mathrm{J}=5.6 \mathrm{~Hz}), 8.08(\mathrm{~d}, 2 \mathrm{H}, \mathrm{J}=6.1 \mathrm{~Hz}), 7.66(\mathrm{bs}, 3 \mathrm{H})$, $7.42(\mathrm{~d}, 2 \mathrm{H}, \mathrm{J}=4.7 \mathrm{~Hz}), 5.88(\mathrm{~s}, 2 \mathrm{H}) \mathrm{ppm}$. MS (70 eV) m/e (rel intensity) 247( $\left.\mathrm{M}^{+}, 1\right), 155(100), 93(6)$. Anal. calcd. for $\mathrm{C}_{17} \mathrm{H}_{15} \mathrm{BF}_{4} \mathrm{~N}_{2}: \mathrm{C}, 61.11 ; \mathrm{H}, 4.52 ; \mathrm{N}, 8.38$. Found: C, 60.81; H, 4.25; N, 8.1. 4-N,N-Dimethylamino-1-(4-pyridylmethyl)pyridinium tetrafluoroborate (9k). Following the method $\mathrm{B}$, to a stirred solution of 1-(4-pyridylmethyl)-2,4,6-triphenylpyridinium tetrafluoroborate $7 \mathrm{c}(0.31 \mathrm{~g}, 0.64 \mathrm{mmol})$ in DMF $(1 \mathrm{ml}), 4-N, N$-dimethylaminopyridine $(0.31 \mathrm{~g}, 2.5 \mathrm{mmol})$ was added, and the mixture was refluxed for $5 \mathrm{~h}$. The usual work-up yielded $9 k(0.6 \mathrm{~g}, 38 \%)$ as white prisms. Mp $170-171^{\circ} \mathrm{C}(\mathrm{EtOH})$. IR: 3067 , $1653,1584,1534,1412,1221,1179,1068 \mathrm{~cm}^{-1} .{ }^{1} \mathrm{H}-\mathrm{NMR}\left(\mathrm{DMSO}_{-} \mathrm{d}_{6}\right) \delta 8.59(\mathrm{~d}, 2 \mathrm{H}, \mathrm{J}=4.4 \mathrm{~Hz}), 8.34(\mathrm{~d}$, $2 \mathrm{H}, \mathrm{J}=7.3 \mathrm{~Hz}), 7.26(\mathrm{~d}, 2 \mathrm{H}, \mathrm{J}=4.7 \mathrm{~Hz}), 7.07(\mathrm{~d}, 2 \mathrm{H}, \mathrm{J}=7.3 \mathrm{~Hz}), 5.44(\mathrm{~s}, 2 \mathrm{H}), 3.19$ (s, 6H) ppm. MS (70 eV) $\mathrm{m} / \mathrm{e}$ (rel intensity) $214\left(\mathrm{M}^{+}, 2\right), 123$ (100), 94 (13). Anal. calcd. for $\mathrm{C}_{13} \mathrm{H}_{16} \mathrm{BF}_{4} \mathrm{~N}_{3}: \mathrm{C}, 51.86 ; \mathrm{H}, 5.36$; $\mathrm{N}, 13.96$; Found: C, 52.09; H, 5.60; N, 13.76 .

1-(4-Pyridylmethyl)isoquinolinium ditetrafluoroborate (91). Following the procedure B, compound 7c (0.93 $\mathrm{g}, 2 \mathrm{mmol})$ and isoquinoline $(1.03 \mathrm{~g}, 0.94 \mathrm{ml}, 8 \mathrm{mmol})$ were refluxed for $6 \mathrm{~h}$. The usual work-up gave $9 \mathrm{i}$ $(0.28 \mathrm{~g}, 35 \%)$ as brownish-yellow prisms. Mp 190-192 ${ }^{\circ} \mathrm{C}(\mathrm{MeOH})$. IR: $3359,3262,3105,1643,1604$, $1514,1400,1055 \mathrm{~cm}^{-1}$. 'H-NMR (DMSO-d 6 ) $\delta 10.2(\mathrm{~s}, 1 \mathrm{H}), 8.83-8.70(\mathrm{~m}, 3 \mathrm{H}), 8.64(\mathrm{~d}, 1 \mathrm{H}, \mathrm{J}=6.6 \mathrm{~Hz})$, $8.51(\mathrm{~d}, 1 \mathrm{H}, \mathrm{J}=8.3 \mathrm{~Hz}), 8.38(\mathrm{~d}, 1 \mathrm{H}, \mathrm{J}=8.3 \mathrm{~Hz}), 8.31(\mathrm{t}, 1 \mathrm{H}, \mathrm{J}=6.8 \mathrm{~Hz}), 8.10(\mathrm{t}, 1 \mathrm{H}, \mathrm{J}=7.4 \mathrm{~Hz}), 7.76(\mathrm{~d}$, $2 \mathrm{H}, \mathrm{J}=5.4 \mathrm{~Hz}), 6.15(\mathrm{~s}, 2 \mathrm{H}) \mathrm{ppm}$. MS $(70 \mathrm{eV}) \mathrm{m} / \mathrm{e}$ (rel intensity) $222\left(\mathrm{M}^{+}, 6\right), 130(100), 94$ (11). Anal. 
calcd. for $\mathrm{C}_{15} \mathrm{H}_{14} \mathrm{~B}_{2} \mathrm{~F}_{8} \mathrm{~N}_{2}$ : C. 45.51: H. 3.56: N. 7.08. Found: C, 45.32; H, 3.46; N, 7.03. Synthesis of 1-(di-pyridin-2-yl methyl)azinium salts.

2,4,6-Triphenyl-1-(Di-pyridin-2-ylmethyl)pyridinium tetrafluoroborate (11). To a stirred suspension of $4 \mathrm{~g}$ (10 mmol) of 2,4.6-triphenylpyrylium tetrafluoroborate 6 in dichloromethane $(50 \mathrm{ml}), 1.9 \mathrm{~g}(10 \mathrm{mmol})$ of (di-pyridin-2-ylmethyl)amine $3 \mathrm{~d}$ and triethylamine $(1.41 \mathrm{ml}, 10 \mathrm{mmol})$ were added. The reaction mixture was stirred at room temperature for $5 \mathrm{~min}$. Then, $1.16 \mathrm{ml}(20 \mathrm{mmol})$ of acetic acid were added, the mixture was stirred for another $15 \mathrm{~min}$, being finally triturated with diethyl ether $(3 \times 10 \mathrm{ml})$ and the ether extracts were separated. The resulting precipitate was filtered and recrystallized yielding $11(1.1 \mathrm{~g}, 20 \%)$ as bright green plates. Mp 193-195 ${ }^{\circ} \mathrm{C}(\mathrm{MeOH})$. IR: $1615,1577,1547,1522,1432,1321,1162,1058 \mathrm{~cm}^{-1}$. ${ }^{\mathrm{l}} \mathrm{H}-\mathrm{NMR}\left(\mathrm{DMSO}_{6}\right) \delta 8.73(\mathrm{~s}, 2 \mathrm{H}), 8.35(\mathrm{~d}, 2 \mathrm{H}, \mathrm{J}=6.4 \mathrm{~Hz}), 7.84(\mathrm{~d}, 2 \mathrm{H}, \mathrm{J}=5.5 \mathrm{~Hz}), 7.69-7.62(\mathrm{~m}, 4 \mathrm{H})$, 7.44-7.20 (m, 12H), 6.53-6.46 (m, 4H) ppm. MS (70 eV) m/e (rel intensity) $476\left(\mathrm{M}^{+}, 11\right), 307(100), 169$ (37), 78 (43). Anal. calcd. for $\mathrm{C}_{34} \mathrm{H}_{26} \mathrm{~N}_{3} \mathrm{BF}_{4}$ : C, 72.48; H, 4.65; N, 7.46. Found: C, 72.78; H, 4.49; N, 7.39

1-(Di-pyridin-2-ylmethyl)pyridinium ditetrafluoroborate (12a). A solution of 1-(di-pyridin-2-ylmethyl)2,4,6-triphenylpyridinium tetrafluoroborate $11(0.2 \mathrm{~g}, 0.35 \mathrm{mmol})$ in $0.9 \mathrm{ml}(10 \mathrm{mmol})$ of pyridine was refluxed for $30 \mathrm{~min}$. Then the mixture was diluted with diethyl ether $(10 \mathrm{ml})$, and the ether layer was separated. The oily residue was treated with ether solution of tetrafluoroboric acid $85 \%(0.07 \mathrm{ml}, 0.35$ mmol) yielding on crystallization 12a $(0.045 \mathrm{~g}, 30 \%)$ as pale yellow prisms. Mp $210-212^{\circ} \mathrm{C}(\mathrm{EtOH})$. IR: 3093, 3022, 2599, 1629, 1482, 1441, $1061 \mathrm{~cm}^{-1}$. ${ }^{1} \mathrm{H}-\mathrm{NMR}\left(\right.$ DMSO-d $_{6}$ ) $\delta 9.24$ (dd, 2H, J= 5.9 and $0.5 \mathrm{~Hz}$ ), $8.67(\mathrm{dt}, 1 \mathrm{H}, \mathrm{J}=7.8$ and $0.8 \mathrm{~Hz}), 8.58(\mathrm{~d}, 2 \mathrm{H}, \mathrm{J}=4.1 \mathrm{~Hz}), 8.18(\mathrm{t}, 2 \mathrm{H}, \mathrm{J}=7.1 \mathrm{~Hz}), 7.94(\mathrm{dt}, 2 \mathrm{H}, \mathrm{J}=7.6$ and $1.2 \mathrm{~Hz}), 7.79(\mathrm{~s}, 1 \mathrm{H}), 7.52-7.46(\mathrm{~m}, 4 \mathrm{H}) \mathrm{ppm}$. MS $(70 \mathrm{eV}) \mathrm{m} / \mathrm{e}$ (rel intensity). 249( $\left.\mathrm{M}^{+}, 7\right), 171(100), 80$ (100). Anal. calcd. for $\mathrm{C}_{16} \mathrm{H}_{15} \mathrm{~B}_{2} \mathrm{~F}_{8} \mathrm{~N}_{3}$ : C, 45.44; $\mathrm{H}, 3.57 ; \mathrm{N}, 9.93$. Found: $\mathrm{C}, 45.59 ; \mathrm{H}, 3.67 ; \mathrm{N}, 9.85$. 4-Phenyl-1-(Di-pyridin-2-ylmethyl)pyridinium tetrafluoroborate (12b) To a suspension of 1-(di-pyridin-2-yl methyl)-2,4,6-triphenylpyridinium tetrafluoroborate $11(0.35 \mathrm{~g}, 0.62 \mathrm{mmol})$ in DMF (2 ml), 4-phenyl pyridine $(0.39 \mathrm{~g}, 2.48 \mathrm{mmol})$ was added, and the reaction mixture was refluxed for $15 \mathrm{~min}$. Then it was diluted with diethyl ether $(10 \mathrm{ml})$, giving a solid which on recrystallization afforded $12 \mathrm{~b}(0.25 \mathrm{~g}, 10 \%)$ as white prisms. Mp 155-156 ${ }^{\circ} \mathrm{C}(\mathrm{EtOH})$. IR: $1636,1589,1471,1436,1161,1064 \mathrm{~cm}^{-1} .{ }^{1} \mathrm{H}-\mathrm{NMR}\left(\mathrm{CD}_{3} \mathrm{OD}\right) \delta$ $9.2(\mathrm{~d}, 2 \mathrm{H}, \mathrm{J}=7.3 \mathrm{~Hz}), 8.63(\mathrm{~d}, 2 \mathrm{H}, \mathrm{J}=6.2 \mathrm{~Hz}), 8.41$ (d, 2H, J=7.3 Hz), 8.03-7.98 (m, 2H), 7.94 (td, 2H, $\mathrm{J}=7.7$ and $1.8 \mathrm{~Hz}), 7.67-7.59(\mathrm{~m}, 3 \mathrm{H}), 7.54-7.45(\mathrm{~m}, 5 \mathrm{H}) \mathrm{ppm}$. MS $(70 \mathrm{eV}) \mathrm{m} / \mathrm{e}$ (rel intensity): $324\left(\mathrm{M}^{+}\right.$, 4), $171(62), 156$ (100). Anal. calcd. for $\mathrm{C}_{22} \mathrm{H}_{18} \mathrm{BF}_{4} \mathrm{~N}_{3}: \mathrm{C}, 64.26 ; \mathrm{H}, 4.41 ; \mathrm{N}, 10.22$. Found: $\mathrm{C}, 64.48 ; \mathrm{H}$, $4.07 ; \mathrm{N}, 10.13$.

1-(Di-pyridin-2-ylmethyl)isoquinolinium ditetrafluoroborate (12c). A mixture of 1-(di-pyridin-2-ylmethyl)2,4,6-triphenylpyridinium tetrafluoroborate $11(0.23 \mathrm{~g}, 0.4 \mathrm{mmol})$ and $0.19 \mathrm{ml}(1.6 \mathrm{mmol})$ of isoquinoline, were refluxed for $30 \mathrm{~min}$. Work-up procedure was followed as for $12 \mathrm{a}$, yielding $12 \mathrm{c}(47 \mathrm{mg}, 25 \%)$ as brown prisms. Mp $166-168^{\circ} \mathrm{C}(\mathrm{EtOH})$. IR: $3419,1644,1588,1471,1435,1398,1153,1062 \mathrm{~cm}^{-1} .{ }^{1} \mathrm{H}-$ 
NMR (DMSO-d $\left.\mathrm{d}_{6}\right) \delta 10.24(\mathrm{~s}, 1 \mathrm{H}), 8.93(\mathrm{dd}, 1 \mathrm{H}, \mathrm{J}=7.1$ and $1.5 \mathrm{~Hz}), 8.64-8.58(\mathrm{~m}, 4 \mathrm{H}), 8.36(\mathrm{~d}, 1 \mathrm{H}, \mathrm{J}=8.0$ $\mathrm{Hz}$ ), 8.28 (ddd, $1 \mathrm{H}, \mathrm{J}=7.9,7.5$ and $1.2 \mathrm{~Hz}$ ), 8.06 (ddd, $1 \mathrm{H}, \mathrm{J}=7.7,7.5$ and $1.2 \mathrm{~Hz}$ ), 7.96 (td, $2 \mathrm{H}, \mathrm{J}=7.8$ and $2.0 \mathrm{~Hz}), 7.89(\mathrm{~s}, 1 \mathrm{H}), 7.55(\mathrm{~d}, 2 \mathrm{H}, \mathrm{J}=7.8 \mathrm{~Hz}), 7.49(\mathrm{dd}, 1 \mathrm{H}, \mathrm{J}=7.6$ and $1.0 \mathrm{~Hz}), 7.48(\mathrm{dd}, \mathrm{J}=7.6$ and $1.0 \mathrm{~Hz}) \mathrm{ppm} . \mathrm{MS}(70 \mathrm{eV}) \mathrm{m} / \mathrm{e}$ (rel intensity): $299\left(\mathrm{M}^{+} .5\right), 171(100), 130(98)$. Anal. calcd. for $\mathrm{C}_{20} \mathrm{H}_{17} \mathrm{~B}_{2} \mathrm{~F}_{8} \mathrm{~N}_{3}$ : C. 50.79; H. 3.62; N, 8.88. Found: C. 50.88; H, 3.97: N, 9.13. Acylation of ylides 10 .

$N$-[2-(4-Chlorobenzoyloxy)-2-(4-chlorophenyl)-1-(2'pyridyl)vinyl]pyridinium chloride (20). To a suspension of $9 \mathrm{a}(0.2 \mathrm{~g}, 0.58 \mathrm{mmol})$ in dichloromethane $(6 \mathrm{ml})$ and aqueous potassiun carbonate $(6 \mathrm{ml}), 4$ chlorobenzoyl chloride $(0.17 \mathrm{ml}, 1.28 \mathrm{mmol})$ was added. The mixture was stirred at room temperature for $30 \mathrm{~min}$. Then, the organic layer was separated and the aqueous one was extracted with dichloromethane $(3 \times 6 \mathrm{ml})$. All the organic extracts were mixed, dried over $\mathrm{Na}_{2} \mathrm{SO}_{4}$, concentrated and recrystallized in water to give $16(42 \mathrm{mg}, 15 \%)$ as white prisms. $\mathrm{Mp} 144-146^{\circ} \mathrm{C}\left(\mathrm{H}_{2} \mathrm{O}\right)$. IR: $1750,1626,1591,1471,1245,1180$, 1078, 1036, $1009 \mathrm{~cm}-1 .{ }^{1} \mathrm{H}-\mathrm{NMR}$ (DMSO-d $\left.{ }_{6}\right) \delta 9.33(\mathrm{~d}, 2 \mathrm{H}, \mathrm{J}=5.7 \mathrm{~Hz}), 8.73(\mathrm{t}, 1 \mathrm{H}, \mathrm{J}=8.25 \mathrm{~Hz}), 8.56(\mathrm{~d}$, $1 \mathrm{H}, \mathrm{J}=4.0 \mathrm{~Hz}$ ), $8.27(\mathrm{t}, 2 \mathrm{H}, \mathrm{J}=6.6 \mathrm{~Hz}), 7.85-7.77(\mathrm{~m}, 3 \mathrm{H}), 7.61-7.52(\mathrm{~m}, 6 \mathrm{H}), 7.43(\mathrm{t}, 1 \mathrm{H}, \mathrm{J}=4.4 \mathrm{~Hz}), 7.29$ (d, 1H, J=7.5 Hz) ppm. MS (70 eV) m/e (rel intensity) $446\left(\mathrm{M}^{+}, 4\right), 308$ (15), 231 (44), 138 (100). Anal. calcd. for $\mathrm{C}_{25} \mathrm{H}_{17} \mathrm{Cl}_{3} \mathrm{~N}_{2} \mathrm{O}_{2}: \mathrm{C}, 62.24 ; \mathrm{H}, 3.55 ; \mathrm{N}, 5.81$. Found: $\mathrm{C}, 62.44 ; \mathrm{H}, 3.78 ; \mathrm{N}, 6.03$.

Reaction with acetylene dipolarophiles.

Method A. To a suspension of the salt 9 a $(0.58 \mathrm{mmol})$, a solution of $50 \%$ aqueous potassiun carbonate (12 $\mathrm{ml})$ and dichloromethane $(6 \mathrm{ml})$, the corresponding acetylene derivative $(0.7 \mathrm{mmol})$ was added, and the mixture was stirred at room temperature for the time indicated. The reaction mixture was extracted with dichloromethane $(3 \times 10 \mathrm{ml})$ and the organic phase dried over $\mathrm{Na}_{2} \mathrm{SO}_{4}$ and evaporated to give a solid residue, which was purified by column chromatography on silica gel using hexane:EtOAc mixture as eluent. Recrystallization gave analytical samples of $\mathbf{2 2 a} \mathbf{a}, \mathbf{b}$.

Method B. A suspension of the salt $9 \mathrm{~b}(0.18 \mathrm{~g}, 0.54 \mathrm{mmol})$, anhydrous potassium carbonate $(0.77 \mathrm{~g}, 5.4$ $\mathrm{mmol})$ and the corresponding acetylene $(0.65 \mathrm{mmol})$ in dichloromethane $(20 \mathrm{ml})$, was stirred at room temperature for $20 \mathrm{~h}$. The inorganic residue was filtered off and washed with dichloromethane $(3 \times 5 \mathrm{ml})$. The organic extracts were concentrated and the residue purified by chromatography on silica gel using dichloromethane as eluent. On crystallization, compounds $22 \mathrm{c}, \mathbf{d}$ were obtained.

3-Pyridin-2-yl indolizine-1-carboxylic acid methyl ester (22a). Following the method A, 9a $(0.2 \mathrm{~g}, 0.58$ mmol) and methyl propiolate $(0.062 \mathrm{ml}, 0.695 \mathrm{mmol})$ were stirred for $30 \mathrm{~min}$. Chromatography using an hexane:EtOAc (9.5:0.5) mixture gave after crystallization, 22a (40 mg, 28\%) as white prisms. Mp 131$132^{\circ} \mathrm{C}$ (EtOH). IR: $1694,1588,1535,1503,1446,1432,1371,1218,1041 \mathrm{~cm}-1 .{ }^{1} \mathrm{H}-\mathrm{NMR}\left(\mathrm{CCl}_{3} \mathrm{D}\right) \delta$ $10.07(\mathrm{~d}, 1 \mathrm{H}, \mathrm{J}=7.3 \mathrm{~Hz}), 8.63(\mathrm{dt}, 1 \mathrm{H}, \mathrm{J}=5.1 \mathrm{~Hz}$ and $1.1 \mathrm{~Hz}), 8.29(\mathrm{dt}, 1 \mathrm{H}, \mathrm{J}=9.1$ and $1.1 \mathrm{~Hz}), 7.75(\mathrm{~s}, 1 \mathrm{H})$, 7.73-7.70 (m, 2H), 7.2-7.1 (m, 2H), $6.87(\mathrm{td}, 1 \mathrm{H}, \mathrm{J}=7 \mathrm{~Hz}, 1.5 \mathrm{~Hz}), 3.93(\mathrm{~s}, 3 \mathrm{H}) \mathrm{ppm}$. MS (70 eV) m/e (rel intensity) $252\left(\mathrm{M}^{+}, 99\right), 221$ (100), 193 (33), $110(41), 89(90), 78$ (21). Anal. calcd. for $\mathrm{C}_{15} \mathrm{H}_{12} \mathrm{~N}_{2} \mathrm{O}_{2}$. 
$1 / 2 \mathrm{H}_{2} \mathrm{O}: \mathrm{C}, 68.95 ; \mathrm{H}, 5.01 ; \mathrm{N}, 10.72$. Found: C. 68.87; H, 4.98; N, 10.72 .

3-Pyridin-2-yl indolizine-1,2-dicarboxylic acid dimethyl ester (22b). Following the method A, 9a (0.1 g, $0.29 \mathrm{mmol})$ in $50 \%$ aqueous potassium carbonate $(6 \mathrm{ml})$ and dichloromethane $(6 \mathrm{ml})$, and DMAD $(0.044$ $\mathrm{ml}, 0.35 \mathrm{mmol})$ were stirred for $1 \mathrm{~h}$. Chromatography using an hexane:EtOAc (8:2) mixture gave, after crystallization. $22 \mathrm{~b}(20 \mathrm{mg}, 23 \%)$ as white prisms. $\mathrm{Mp} 137-138^{\circ} \mathrm{C}\left(\mathrm{EtOH}-\mathrm{H}_{2} \mathrm{O}\right) . \mathrm{IR}: 1723,1702,1686$, $1584,1510,1448,1259,1233,1177 \mathrm{~cm}-1 .{ }^{1} \mathrm{H}-\mathrm{NMR}\left(\mathrm{CCl}_{3} \mathrm{D}\right) \delta 9.22(\mathrm{~d}, 1 \mathrm{H}, \mathrm{J}=7.1 \mathrm{~Hz}), 8.72(\mathrm{~d}, 1 \mathrm{H}, \mathrm{J}=4.2$ $\mathrm{Hz}), 8.26(\mathrm{~d}, 1 \mathrm{H}, \mathrm{J}=8.9 \mathrm{~Hz}), 7.77(\mathrm{td}, 1 \mathrm{H}, \mathrm{J}=8$ and $1.8 \mathrm{~Hz}), 7.61(\mathrm{~d}, 1 \mathrm{H}, \mathrm{J}=8 \mathrm{~Hz}), 7.3-7.1(\mathrm{~m}, 2 \mathrm{H}), 6.84(\mathrm{t}$, $1 \mathrm{H}, \mathrm{J}=7.1 \mathrm{~Hz}), 3.91(\mathrm{~s}, 6 \mathrm{H}) \mathrm{ppm}$. MS (70 eV) m/e (rel. intensity) $310\left(\mathbf{M}^{+}, 100\right), 279(75), 221(34), 193$ (27), 192 (54), 78 (32). Anal. calcd. for $\mathrm{C}_{17} \mathrm{H}_{14} \mathrm{~N}_{2} \mathrm{O}_{4} \cdot 1 / 2 \mathrm{H}_{2} \mathrm{O}$ : C, 63.94; H. 4.69; N, 8.76. Found: C $63.33, \mathrm{H} 4.99, \mathrm{~N} 9.00$.

7-Phenyl-3-pyridin-2-yl indolizine-1-carboxylic acid methyl ester (22c). Following the method B, $9 \mathbf{b}$ and methyl propiolate $(0.06 \mathrm{ml}$., $0.65 \mathrm{mmol})$ were stirred, and $80 \mathrm{mg}(45 \%)$ of $22 \mathrm{c}$ were isolated as white

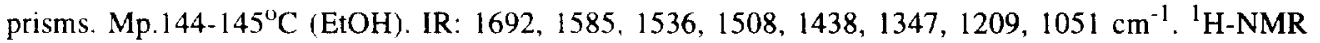
$\left(\mathrm{CCl}_{3} \mathrm{D}\right) \delta 10.12(\mathrm{~d}, 1 \mathrm{H}, \mathrm{J}=8.4 \mathrm{~Hz}), 8.64(\mathrm{~d}, 1 \mathrm{H}, \mathrm{J}=4.7 \mathrm{~Hz}), 8.55(\mathrm{~s}, 1 \mathrm{H}), 7.78-7.71(\mathrm{~m}, 5 \mathrm{H}), 7.51-7.37(\mathrm{~m}$, $3 \mathrm{H}), 7.19-7.12(\mathrm{~m}, 2 \mathrm{H}), 3.94(\mathrm{~s}, 3 \mathrm{H}) \mathrm{ppm}$. MS (70 Ev) m/e (rel intensity) $328\left(\mathrm{M}^{+}, 82\right), 297(47), 165$ (100), 148 (62), 78 (20). Anal. calcd. for $\mathrm{C}_{21} \mathrm{H}_{16} \mathrm{~N}_{2} \mathrm{O}_{2}: \mathrm{C}, 76.81 ; \mathrm{H}, 4.91 ; \mathrm{N}, 8.53$. Found: C, 77.18; $\mathrm{H}$, 4.96: N, 8.55 .

7-Phenyl-3-pyridin-2-yl indolizine-1,2-dicarboxylic acid dimethyl ester (22d). Following the method B, 9b and DMAD $(0.08 \mathrm{ml}, 0.65 \mathrm{mmol})$ were stirred, and $70 \mathrm{mg}(34 \%)$ of $22 \mathrm{~d}$ were isolated as white prisms. Mp $139-140^{\circ} \mathrm{C}(\mathrm{MeOH})$. IR: $1735,1688,1584,1513,1449,1383,1240,1209,1172,1135 \mathrm{~cm}^{-1} .{ }^{1} \mathrm{H}-\mathrm{NMR}$ $\left(\mathrm{CCl}_{3} \mathrm{D}\right) \delta 9.32(\mathrm{~d}, 1 \mathrm{H}, \mathrm{J}=7.3 \mathrm{~Hz}), 8.73(\mathrm{~d}, 1 \mathrm{H}, \mathrm{J}=4 \mathrm{~Hz}), 8.51(\mathrm{~s}, 1 \mathrm{H}), 7.79-7.61(\mathrm{~m}, 4 \mathrm{H}), 7.52-7.4(\mathrm{~m}$, $3 \mathrm{H}), 7.26(\mathrm{~s}, \mathrm{lH}), 7.13(\mathrm{~d}, 1 \mathrm{H}, \mathrm{J}=7 \mathrm{~Hz}), 3.96(\mathrm{~s}, 6 \mathrm{H}) \mathrm{ppm}$. MS $(70 \mathrm{eV}) \mathrm{m} / \mathrm{e}$ (rel intensity) $386\left(\mathrm{M}^{+}, 100\right)$; 355 (51); 268 (45): $165(60) ; 78$ (30). Anal. calcd. for $\mathrm{C}_{23} \mathrm{H}_{18} \mathrm{~N}_{2} \mathrm{O}_{4}: \mathrm{C}, 71.49 ; \mathrm{H}, 4.70 ; \mathrm{N}, 7.25$. Found: $\mathrm{C}$, $71.37 ; \mathrm{H}, 5.00 ; \mathrm{N}, 7.36$.

\section{Reaction with carbon disulfide/methyl iodide.}

1-[2:2 -Bis(methylthio)-1'-(2'-pyridyl-vinyl)]pyridinium Iodide (25). To a stirred suspension of $9 \mathrm{a}(0.7 \mathrm{~g}$, $2 \mathrm{mmol})$ in $50 \%$ aqueous potassium carbonate $(20 \mathrm{ml})$, carbon disulphide $(20 \mathrm{ml})$ and methyl iodide $(0.5$ $\mathrm{ml}, 8 \mathrm{mmol}$ ) were added, and the mixture was stirred for $20 \mathrm{~h}$. The precipitate was filtered and washed with water until neutral. Recrystallization gave $25(0.32 \mathrm{~g}, 40 \%)$ as yellow prisms. Mp $160-161^{\circ} \mathrm{C}(\mathrm{EtOH})$. IR: 3433, 3009, 1621, 1581, 1538, 1464, $1425 \mathrm{~cm}^{-1}$. ' $\mathrm{H}-\mathrm{NMR}$ (DMSO-d 6 ) $\delta 9.26$ (d, 2H, J= 6.8 Hz), 8.78 (t. $1 \mathrm{H}, \mathrm{J}=7.9 \mathrm{~Hz}), 8.50(\mathrm{~d}, 1 \mathrm{H}, \mathrm{J}=4.2 \mathrm{~Hz}), 8.29(\mathrm{t}, 2 \mathrm{H}, \mathrm{J}=6.8 \mathrm{~Hz}), 8.0-7.95(\mathrm{~m}, 2 \mathrm{H}), 7.41(\mathrm{dd}, 1 \mathrm{H}, \mathrm{J}=6.1$ and $1.5 \mathrm{~Hz}), 2.46$ (s. 3H), $2.39(\mathrm{~s}, 3 \mathrm{H}) \mathrm{ppm}$. MS (70 eV) m/e (rel intensity): $276\left(\mathrm{M}^{+}, 2\right), 245(58), 198$ (28), 80 (100). Anal. calcd. for $\mathrm{C}_{14} \mathrm{H}_{15} \mathrm{IN}_{2} \mathrm{~S}_{2}$ : C, 41.79; H, 3.75; N, 6.96. Found: C, 41.74; H, 3.66; N, 6.88

Reaction with isothiocyanates. General procedure. To a suspension of the corresponding $9(0.58 \mathrm{mmol})$ in 
$50 \%$ aqueous potassiun carbonate $(5 \mathrm{ml})$ and dichloromethane $(5 \mathrm{ml})$, phenyl isothiocyanate $(0.64 \mathrm{mmol})$ was added and the mixture was stirred at room temperature. The organic layer was separated and the aqueous layer was extracted with dichloromethane $(3 \times 10 \mathrm{ml})$. The combined organic extracts were dried over $\mathrm{Na}_{2} \mathrm{SO}_{4}$ and evaporated, to give a residue which was purified by chromatography on silica gel using a mixture $\mathrm{CH}_{2} \mathrm{Cl}_{2}$ :acetone $(9: 1)$ as eluent, to give the betaines $27 \mathbf{a}, \mathbf{b}$.

1-Phenyl-3-(pyridin-2-vl)imidazo(1,2-a]pyridimium-2-thiolate (27a). Following the general procedure, 9a $(0.2 \mathrm{~g}, 0.58 \mathrm{mmol})$ and phenyl isothiocyanate $(0.08 \mathrm{mml}, 0.64 \mathrm{mmol})$ were stirred for $3 \mathrm{~h}$. After the usual work-up, the betaine $27 \mathrm{a}$ was isolated (45 $\mathrm{mg}, 25 \%$ ) as yellow prisms. Mp 190-191 ${ }^{\circ} \mathrm{C}$. IR: 1583, 1491, $1431,1337,1299,1272,1230,1144,1109 \mathrm{~cm}^{-1} .{ }^{1} \mathrm{H}-\mathrm{NMR}\left(\mathrm{DMSO}-\mathrm{d}_{6}\right) \delta 10.21$ (d, $\left.1 \mathrm{H}, \mathrm{J}=6.8 \mathrm{~Hz}\right), 9.50$ $(\mathrm{d}, 1 \mathrm{H}, \mathrm{J}=8.3 \mathrm{~Hz}), 8.68(\mathrm{~d}, 1 \mathrm{H}, \mathrm{J}=5.9 \mathrm{~Hz}), 7.88(\mathrm{dt}, 1 \mathrm{H}, \mathrm{J}=7.6$ and $2.0 \mathrm{~Hz}), 7.66-7.49(\mathrm{~m}, 6 \mathrm{H}), 7.37(\mathrm{dt}$, $1 \mathrm{H}, \mathrm{J}=7.1$ and $1.2 \mathrm{~Hz}), 7.26(\mathrm{ddd}, 1 \mathrm{H}, \mathrm{J}=5.9,4.9$ and $1.2 \mathrm{~Hz}), 7.11(\mathrm{~d}, 1 \mathrm{H}, \mathrm{J}=8.8 \mathrm{~Hz}) \mathrm{ppm} . \mathrm{MS}(70 \mathrm{eV})$ m/e (rel intensity) $303\left(\mathrm{M}^{+}, 100\right), 225$ (25), 78 (50). Anal. calcd. for $\mathrm{C}_{18} \mathrm{H}_{13} \mathrm{~N}_{3} \mathrm{~S}, 1 / 2 \mathrm{H}_{2} \mathrm{O}: \mathrm{C}, 69.43 ; \mathrm{H}$, $4.53 ;$ N, 13.49. Found: C, 68.98; H, 4.67; N. 13.69.

I-(4-Chlorophenyl)-3-(pyridin-2-yl)imidazoll,2-a]pyridinium-2-thiolate (27b). Following the general procedure, $9 \mathbf{a}(0.2 \mathrm{~g}, 0.58 \mathrm{mmol})$ was reacted with p-chlorophenyl isothiocyanate $(0.12 \mathrm{~g}, 0.7 \mathrm{mmol})$ for 5 h. After the usual work-up, the betaine $27 \mathrm{~b}$ was isolated (34 $\mathrm{mg}, 18 \%$ ) as yellow prisms. $\mathrm{Mp} 230-231^{\circ} \mathrm{C}$ (EtOH). IR: $1581,1490,1430,1334,1303,1274,1233,1086 \mathrm{~cm}^{-1} .{ }^{1} \mathrm{H}-\mathrm{NMR}\left(\mathrm{CCl}_{3} \mathrm{D}\right) \delta 10.36(\mathrm{~d}, 1 \mathrm{H}, \mathrm{J}=7$ $\mathrm{Hz}), 9.56(\mathrm{~d}, 1 \mathrm{H}, \mathrm{J}=8.4 \mathrm{~Hz}), 8.65(\mathrm{dd}, 1 \mathrm{H}, \mathrm{J}=4$ and $1.1 \mathrm{~Hz}), 7.85(\mathrm{td}, 1 \mathrm{H}, \mathrm{J}=7.7$ and $1.8 \mathrm{~Hz}), 7.6(\mathrm{~d}, 2 \mathrm{H}$, $\mathrm{J}=8.8 \mathrm{~Hz}), 7.5-7.4(\mathrm{~m}, 3 \mathrm{H}), 7.19(\mathrm{t}, 2 \mathrm{H}, \mathrm{J}=5.5 \mathrm{~Hz}), 7.06(\mathrm{~d}, 1 \mathrm{H}, \mathrm{J}=8.8 \mathrm{~Hz}) \mathrm{ppm} . \mathrm{MS}(70 \mathrm{eV}) \mathrm{m} / \mathrm{e}$ (rel. intensity) $337\left(\mathrm{M}^{+}, 100\right), 226(18), 150(47), 78$ (33). Anal. calcd. for $\mathrm{C}_{18} \mathrm{H}_{12} \mathrm{ClN}_{3} \mathrm{~S} .1 \mathrm{H}_{2} \mathrm{O}: \mathrm{C}, 60.58 ; \mathrm{H}$, $3.95 ; \mathrm{N}, 11.77$. Found: $\mathrm{C}, 60.81 ; \mathrm{H}, 3.88 ; \mathrm{N}, 11.62$.

1,7-Diphenyl-3-(py ridin-2-yl)imidazoll,2-alpyridinum-2-thiolate $(27 \mathrm{c})$. To a suspension of $9 \mathbf{b}(0.16 \mathbf{g}, 0.48$ mmol), and anhidrous potassiun carbonate $(0.68 \mathrm{~g} .4 .8 \mathrm{mmol})$ in dichloromethane $(15 \mathrm{ml})$, phenyl isothiocyanate $(0.07 \mathrm{ml}, 0.58 \mathrm{mmol})$ was added. The mixture was stirred for $20 \mathrm{~h}$. After the usual work-up, recrystallization yielded $60 \mathrm{mg}(33 \%)$ of $27 \mathrm{c}$ as yellow prisms. Mp $238-240^{\circ} \mathrm{C}$ (EtOH). IR: 1585,1486 , $1455,1431,1338,1303,1274,1249 \mathrm{~cm}^{-1}$. ${ }^{1} \mathrm{H}-\mathrm{NMR}\left(\mathrm{DMSO}_{6}\right) \delta 10.3(\mathrm{~d}, 1 \mathrm{H}, \mathrm{J}=8 \mathrm{~Hz}), 9.56(\mathrm{~d}, 1 \mathrm{H}, \mathrm{J}=8$ $\mathrm{Hz}), 8.72-8.69(\mathrm{~m}, 1 \mathrm{H}), 7.94-7.86(\mathrm{~m}, 1 \mathrm{H}), 7.80-7.72(\mathrm{~m}, 3 \mathrm{H}), 7.67-7.55(\mathrm{~m}, 5 \mathrm{H}), \quad 7.51-7.44(\mathrm{~m}, 3 \mathrm{H})$, $7.31-7.25(\mathrm{~m}, 1 \mathrm{H}), 7.21(\mathrm{~d}, 1 \mathrm{H}, \mathrm{J}=1.5 \mathrm{~Hz}) \mathrm{ppm}$. MS (70 eV) m/e (rel intensity) $379\left(\mathrm{M}^{+}, 100\right), 378(99)$, 302 (14), 301 (20), 78 (47). Anal. calcd. for $\mathrm{C}_{24} \mathrm{H}_{17} \mathrm{~N}_{3} \mathrm{~S}: \mathrm{C}, 75.96 ; \mathrm{H}, 4.52 ; \mathrm{N}, 11.02 ; \mathrm{S}, 8.44$. Found: C, $75.85 ; \mathrm{H}, 4.27 ; \mathrm{N}, 10.88 ; \mathrm{S}, 8.21$.

\section{ACKNOWLEDGEMENTS}

The authors wish to express their thanks to the Comision Interministerial de Ciencia y Tecnologia (C.I.C.Y.T) for financial support (Project PB90-0284), and to the Ministerio de Educación y Ciencia by a 
grant to one of them (A.M.C.).

\section{REFERENCES}

1. Ollis, W. D.: Stanford, S. P.; Ramsden, C. A. Tetrahedron 1985, 41, 2239-2329.

2. Sliva, W. Hetcrocycles 1991, 32, 22412273 and references cited therein.

3. Surpateanu. G.; Lablanche-Combier, L. Heterocycles 1984, 22, 2079-2128.

4. a) Katritzky, A.R.; Motherhack, D. J. Chem. Soc., Perkin trans.I 1976, 909-912. b) Alcalde, E.; Perez-Garcia, L.; Miravitlles, C.; Rius. J.; Valenti, E. I. Org. Chem. 1992, 57, 4829-4838.

5. a) Cuadro, A. M.: Alvarez-Builla, J.: Vaquero, J. J. Heterocycles, 1988, 28, 1233-1240. b) Cuadro, A. M.; Alvarez-Builla, J.; Vaquero, J. J.; Heterocycles, 1989, 29, 57-65. c) Carceller, R.; GarciaNavio, J. L.; Izquierdo, M. L.; Alvarez-Builla, J.; Sanz-Aparicio, J.; Florencio, F. Heterocycles, 1989, 29, 1877-1889. d) Molina, A.; Cuadro, A. M.; Alvarez-Builla, J.; Vaquero, J. J.; GarciaNavio, J. L. Heterocycles, 1990, 3/, 145I-1458. e) Cuadro, A. M.; Novella, J. L.; Molina, A.; Alvarez-Builla, J.; Vaquero, J. J. Tetrahedron, 1990, 46, 6033-6046.

6. a) Carceller, R.; Garcia-Navio, J. L.; Izquierdo, M. L.; Alvarez-Builla, J. Tetrahedron Lett. 1993, 34, 2019-2020. b) Carceller, R.; Garcia-Navio, J. L.; Izquierdo, M. L.; Alvarez-Builla, J. Tetrahedron 1994, 50, 4995-5012. c) Beyer, H.; Thieme, E. J. Prakt. Chem. 1966, 31, 293-303.

7. Genisson, Y.: Marazano, C.; Mehmandoust, M.; Gnecco, D.; Das, B. C. Synlett. 1992, 431-434.

8. a) Katritzky, A. R.; Marson, C. M. Angew. Chem. Int. Ed. Engl. 1984, 23, 420-429. b) Katritzky, A. R.: Bapat, J. B.; Blade, R. J.: Leddy, B. P.; Nie, P. L.; Ramsden, C. A.; Thind. S. S. J. Chem. Soc. Perk in Trans. 1 1979, 418-425. The starting 1-(pyridylmethyl)triphenylpyridinium

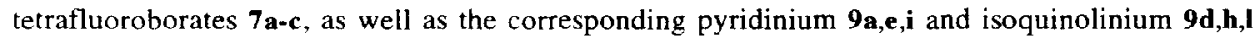
derivatives were reported as perchlorates in this reference).

9. Katritzky, A.R. Tetrahedron 1980, 36. 679-699.

10. Cuadro, A. M.; Alvarez-Builla, J.; Vaquero, J. J. Heterocycles 1988, 27, 1233-1240.

11. a) Gandasegui, M. T.; Alvarez-Builla. J. Heterocycles 1990, 31, 1801-1809. b) Cuadro, A. M.; Novella, J. L.; Molina, A.: Alvarez-Builla, J.; Vaquero, J. J. Tetrahedron 1990, 46, 6033-6046, and literature cited therein.

12. Minguez, J. M.: Gandasegui, M. T.: Vaquero, J. J.; Alvarez-Builla, J.; Garcia-Navio, J. L.; Gago, F.; Ortiz, A. R.; Gomez-Sal, P.; Torres, R.; Rodrigo, M. M. J. Org. Chem. 1993, 58, 6030-6037.

13. Stewart, J. J. P. MOPAC 6.0 A General Molecular Orbital Package. QCPE 455, Quantum Chemistry Program Exchange, Bloomington, IN, 1990.

14. SPARTAN 3.1, Wavefunction, Inc., Irvinc, CA, 1994.13.

15. Awartani, R.; Sakizadeh, K; Gabrielsen, B. J. Chem. Educ. 1986, 172. 